\title{
Atmospheric Pressure Plasma Surface Modification of Titanium for High Temperature Adhesive Bonding
}

\author{
M. Akram ${ }^{1}$, K.M.B.Jansen ${ }^{1}$, L.J.Ernst ${ }^{1}$ and S. Bhowmik ${ }^{2}$ \\ ${ }^{1}$ PME Department, Faculty of Mechanical, Maritime and Materials Engineering \\ ${ }^{2}$ Faculty of Aerospace Engineering \\ Delft University of Technology, Mekelweg 2, 2628 CD Delft, the Netherlands
}

\begin{abstract}
:
In this investigation surface treatment of titanium is carried out by plasma ion implantation under atmospheric pressure plasma in order to increase the adhesive bond strength. Prior to the plasma treatment, titanium surfaces were mechanically treated by sand blasting. It is observed that the contact angle of de-ionized water decreases with the grit blast treatment time. Optical microscopy and Scanning Electron Microscopic (SEM) analysis of untreated and atmospheric plasma treated titanium are carried out to examine the surface characteristics. A substantial improvement in the surface energy of titanium is observed after the atmospheric pressure plasma treatment. The surface energy increases with increasing exposure time of atmospheric pressure plasma. The optimized time of plasma treatment suggested in this investigation results in maximum adhesive bond strength of the titanium. Unmodified and surface modified titanium sheets by atmospheric pressure plasma were adhesively bonded by high temperature resistant polyimide adhesive. The glass transition temperature of this adhesive is $310{ }^{\circ} \mathrm{C}$ and these adhesively bonded joints were cured at high temperature. A substantial improvement in adhesive bond strength was observed after atmospheric pressure plasma treatment.
\end{abstract}

Key Words: Titanium, Surface Modification, Atmospheric Pressure Plasma, Surface Energy, Adhesive Bonding

Corresponding Author:

M.Akram, Tel: +31(0)152789979, FAX: +31(0)152782150

E-mail address:M.Akram@tudelft.nl 


\section{Introduction}

Titanium has many desirable features in terms of high temperature properties, excellent strength to weight ratio, and good corrosion resistance [1]. Therefore, presently, titanium is one of the most effective materials for structural application of spacecraft and aviation. Titanium alloys are widely used in solid rocket booster cases, guidance control pressure vessel and other different applications demanding light weight and reliability. Titanium alloys are extensively employed in solid rocket booster cases, guidance control pressure vessels and a wide variety of applications demanding light weight and reliability[2].

The aerospace industry is also a larger market for titanium products primarily due to exceptional strength to weight ratio, high resistance to elevated temperatures and corrosion [3].Now a days adhesive bonding is the preferred joining technique for titanium structures, where as in the past focus was more on welding and riveting. There are numerous advantages for this technology, such as continuity of adhesive bond which allows more uniform distribution of stresses in the bonded area and the fact that most commonly used titanium alloys demonstrate poor weldability. However, for the adhesive bonding the surface treatment appears to be the critical factor in order to generate a good bond strength and long term durability[4].

The principle objective of this study is to improve the adhesive bond strength of titanium sheets which are bonded together using high temperature resistant polyimide adhesive. For improving the performance of adhesive bonding of titanium for aerospace and space climatic conditions, it is necessary to modify the surface of titanium[5]. The main area of environmental attack on a polymer metal bond is at or close to the adhesive or primer/oxide interface resulting in adhesive failure. To prevent adhesive failure, it is desirable to transfer the locus of failure from the adhesive/adherent interface to within the adhesive, explicitly to cohesive failure[6].

It is reported that for titanium alloy such as TiAl6V4, typical treatment methods are mechanical, chemical or even electrochemical [7].The frequently used method is chromic 
$8 / 22 / 2011$

acid anodizing [8]. Surface roughening followed by plasma ion implantation technology has emerged as a promising alternative solution for the surface treatment of titanium, since it is simple and environment friendly [7].

The properties of oxide films on metals are important in a number of surface phenomena, such as adsorption, localized corrosion and adhesion of organic coatings [9].The surface treatment leads to changes in the oxide chemistry resulting in a direct influence on the subsequent bonding behavior with organic functional groups [10].

Based on this consideration, this study investigates surface modification phenomena of titanium by mechanical treatment under grit blasting followed by atmospheric pressure plasma treatment. Characterization of titanium surface after grit blasting is carried out by contact angle measurement. Surface roughness of the grit blasted samples was measured by surface roughness profiler. Physico-chemical characterization of surface modified titanium was carried out by surface energy measurement and SEM studies. Finally, the lap shear tensile strength is carried out to measure adhesive bond strength of surface modified titanium.

\section{Experimental}

\subsection{Materials}

In this investigation, a titanium alloy Ti 6Al4V of AMS4911 Grade 5 supplied by AmEuro Metals BV Netherlands is used. The chemical composition of this titanium alloy according to chemical composition test result by manufacturer is given in Table 1.

\section{Table 1}

Chemical composition of the TiAl6V4 alloy

\begin{tabular}{|c|c|c|c|c|c|c|c|c|}
\hline Element & Ti & Al & V & Fe & O & C & N & H \\
\hline Weight (\%) & 88.105 & 6.75 & 4.50 & 0.25 & 0.20 & 0.08 & 0.05 & 0.015 \\
\hline
\end{tabular}

High temperature resistant, thermally stable polyimide adhesive \#124-41with Phenylenediamine as its main component is supplied by Creative materials Inc. USA and is 
used for adhesive bonding of Titanium alloy. The glass transition temperature of this polyimide adhesive is above $250{ }^{\circ} \mathrm{C}$ and the service temperature ranges from $-55{ }^{0} \mathrm{C}$ to 250 ${ }^{0} \mathrm{C}$. It is thermal stability at temperatures up to $325^{\circ} \mathrm{C}$. The listed tensile shear strength for this adhesive is 8.6 MPa.

Two test liquids with known surface energy, de-ionized water and formamide were used to determine the polar and dispersion components of surface energies of titanium through measurement of contact angle by the sessile drop method. In this investigation the following three types of titanium samples were studied:

(i) As received titanium Samples

(ii) Grit blasted titanium Samples using alumina grits of mean size 50 micron

(iii) Grit blasted titanium modified under atmospheric pressure plasma

Prior to modification of titanium, the substrates were sequentially cleaned in acetone, methyl alcohol and de-ionized water by an ultrasonic cleaning method.

\subsection{Mechanical Treatment}

The mechanical treatment was carried out in order to increase the macro roughness of titanium by using alumina grit (Edel korund white) in a grit blasting chamber. The alumina grits bag with mean grain size of 150 micron was supplied by Unicorn b.v. The apparatus used was a Unicorn ICS b.v cabinet, rectangular in shape and has a selfcontained, sealed glove box for powder recycling. The spray gun consists of a $708 \mathrm{lpm}$ carbide nozzle and a $708 \mathrm{lpm}$ air jet housed in a large bronze gun. A blasting angle of $90^{\circ}$ was used during treatment. The grit blasting device was operated manually. To ensure repeatability and consistency in the grit blasting operation, the time spent for blasting substrate was selected as 30, 60, 90,120and 150 seconds. Five samples were prepared for each treatment time. After Grit blasting, ultrasonic washing of samples with acetone and ethanol was carried out to remove any loose particles of alumina grit. 


\subsection{Atmospheric Pressure Plasma Treatment}

Atmospheric pressure plasma treatment was carried out on grit blasted and ultrasonically cleaned titanium surfaces. Titanium samples were exposed to atmospheric pressure plasma under a TIGRES Plasma-BLASTER MEF. In this investigation, the treatment distance of substrate from nozzle head of plasma equipment was $10 \mathrm{~mm}$ and the gas used for treatment was air with a total flow rate of $51 \mathrm{l} / \mathrm{min}$ at a pressure of 4.5 bars. The plasma surface treatment time was gradually increased from 5 minutes to 15 minutes in order to find lowest contact angle with increasing time of exposure.

\subsection{Contact Angle Measurement and Surface Energy Estimation}

The surface energy and its polar and dispersion components were calculated using contact angle measurements. Contact angles were measured using a Modular “CAM 200Optical contact angle and surface tension meter” with de-ionized water and formamide as liquid. The surface energy and its polar and dispersion components for all samples were calculated using the following equation [11]

$$
(1+\cos \theta) \gamma_{L V}=2\left(\gamma_{S}^{D} \gamma_{L V}^{D}\right)^{1 / 2}+2\left(\gamma_{S}^{P} \gamma_{L V}^{P}\right)^{1 / 2}
$$

In equation (1), $\theta$ is the measured contact angle of liquid with the solid surface, $\gamma_{L V}$ is the total surface tension of the liquid, $\gamma_{L V}^{D}$ is the dispersion component of the liquid and $\gamma_{L V}^{P}$ is the polar component of the liquid. The total surface tension of the liquids ' $\gamma_{L V}$ ' and their polar $\gamma_{L V}^{P}$ and dispersion $\gamma_{L V}^{D}$ components are known and listed in table 2[12].

Table 2

Polar, dispersion and total surface energy of test liquids

\begin{tabular}{|c|c|c|c|}
\hline Liquids & $\begin{array}{c}\gamma_{L V}^{P} \\
(\mathrm{~m} \mathrm{~N} / \mathrm{m})\end{array}$ & $\begin{array}{c}\gamma_{L V}{ }^{D} \\
(\mathrm{~m} \mathrm{~N} / \mathrm{m})\end{array}$ & $\begin{array}{c}\gamma_{L V} \\
(\mathrm{~m} \mathrm{~N} / \mathrm{m})\end{array}$ \\
\hline Deionized Water & 50.2 & 22.0 & 72.2 \\
\hline Formamide & 18.6 & 39.6 & 58.2 \\
\hline
\end{tabular}


Contact angles of both liquids on the substrate surfaces were determined by the sessile drop method and then the dispersion and polar components of the solid surface were determined. Finally, the total surface energy $\gamma_{S}$ was estimated by adding $\gamma_{S}^{P}$ and $\gamma_{S}^{D}$ as given in Equation (2):

$$
\gamma_{s}=\gamma_{s}^{D}+\gamma_{s}^{P}
$$

\subsection{Surface Roughness Measurements}

Surface roughness of untreated, mechanically treated and mechanically treated followed by atmospheric pressure plasma treated titanium samples was measured using a Wyko NT 3000, surface roughness Profiler. With Wyko profiler, surface roughness can be measured with out contacting it.

\subsection{Adhesive Joint Preparation and Tensile Lap Shear Testing}

Tensile lap shear tests were performed using the standard ASTM D-1002-01. Rectangular specimens, having dimensions 100 x 25 x $3.17 \mathrm{~mm}^{3}$, were used for tensile lap shear testing. The tensile lap shear specimens were prepared by applying the polyimide adhesive. The overlap area was controlled to $25 \times 12.5 \mathrm{~mm}^{2}$. Any excessive adhesive present at the interface was expelled by mechanical pressing of the joint, which resulted in a joint having an adhesive thickness of about $0.20 \mathrm{~mm}$. The adhesive thickness was controlled by adding 1 weight percent of glass beads (200 $\mu$ m diameter) in the adhesive and applying a uniform pressure on all the adhesive bonded joints. Pressure was applied to the lap joint during the curing cycle by two binder clips. The bonded specimens were cured at $175{ }^{0} \mathrm{C}$ for 1 hour with a subsequent post curing at $210{ }^{\circ} \mathrm{C}$ for five hours. Lap Shear testing was carried out using a computer-controlled testing machine, Zwick 250KN static test machine, and a load cell of $100 \mathrm{kN}$. The specimens were loaded in tension at a test speed of $2 \mathrm{~mm} / \mathrm{min}$. five specimens were used for each material. All tests were performed at $22{ }^{0} \mathrm{C}$ and at 50\% humidity. 


\subsection{Microscopic Studies of Substrate Surfaces and Fractrography}

Scanning electron microscopy was used to study the surface texture of titanium samples before and after treatment. Fracture surface of the adhesive bonded joint after lap shear test was also analyzed by scanning electron microscopy. Images were obtained using a JEOL JSM-7500F field emission scanning electron microscope (FE-SEM).

\section{Results}

\subsection{Contact Angle on Surface Modified Titanium}

Contact angle measurements of de-ionized water and form amide after 30, 60, 90, 120,150 seconds grit blasted titanium surfaces are shown in figure 1 . The figure reveals that the contact angle decreases with increasing treatment time of grit blasting and appears to be reach minimum level of 25 degree with de ionized water, and 14 degrees with form amide after 120 sec of grit blasting.

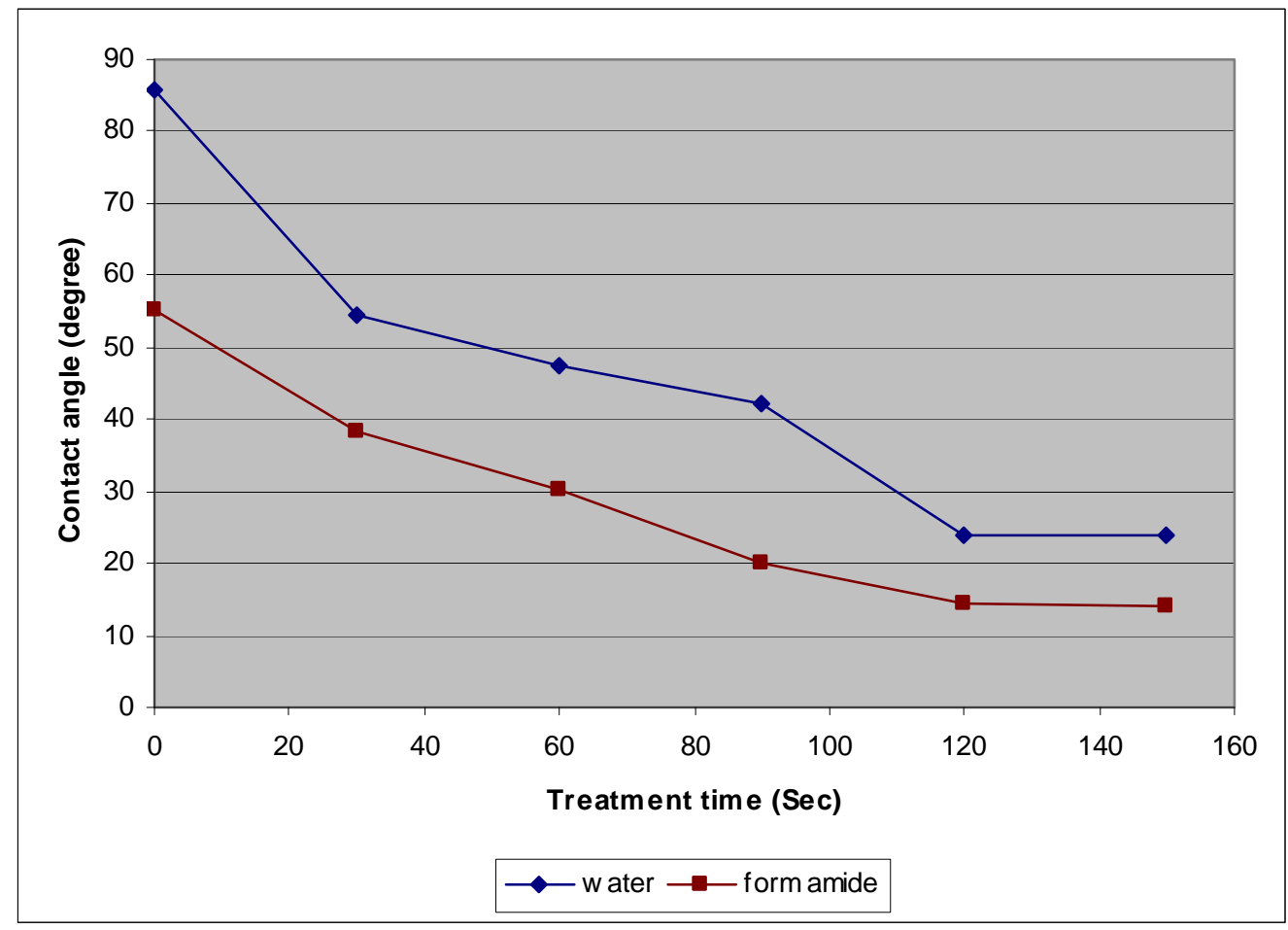

Fig. 1 Contact angle measurement with Deionized water and form amide after mechanical treatment 
These mechanical treated samples were subjected to atmospheric pressure plasma treatment. Figure 2 reveals that contact angle measurements with de-ionized water and form amide decreases from the grit blasted level of 25 degree to 8 degree and in case of form amide from 14 degrees to a much lower value of 2 degrees during atmospric pressure plasma treatment. Actually, when placing a water droplet on the surface directly after plasma cleaning a total wetting was observed. Due to time required for surface tension measurement, a some what larger value of contact angle is measured. It is further evident from the figure that under atmospheric pressure plasma the optimum treatment time is 15 minutes.

The contact angle of de-ionized water and formamide on as received titanium after washing with acetone is $85.6^{\circ}$ and $55.2^{\circ}$ respectively. After $120 \mathrm{sec}$ of grit blasting, these contact angles reduces to $23.9^{\circ}$ and $14.3^{\circ}$. It is interesting to note that after atmospheric pressure plasma treatment of 15 minutes, these values further decreases to $8.1^{\circ}$ and $3.0^{\circ}$ respectively as evident from figure 2.

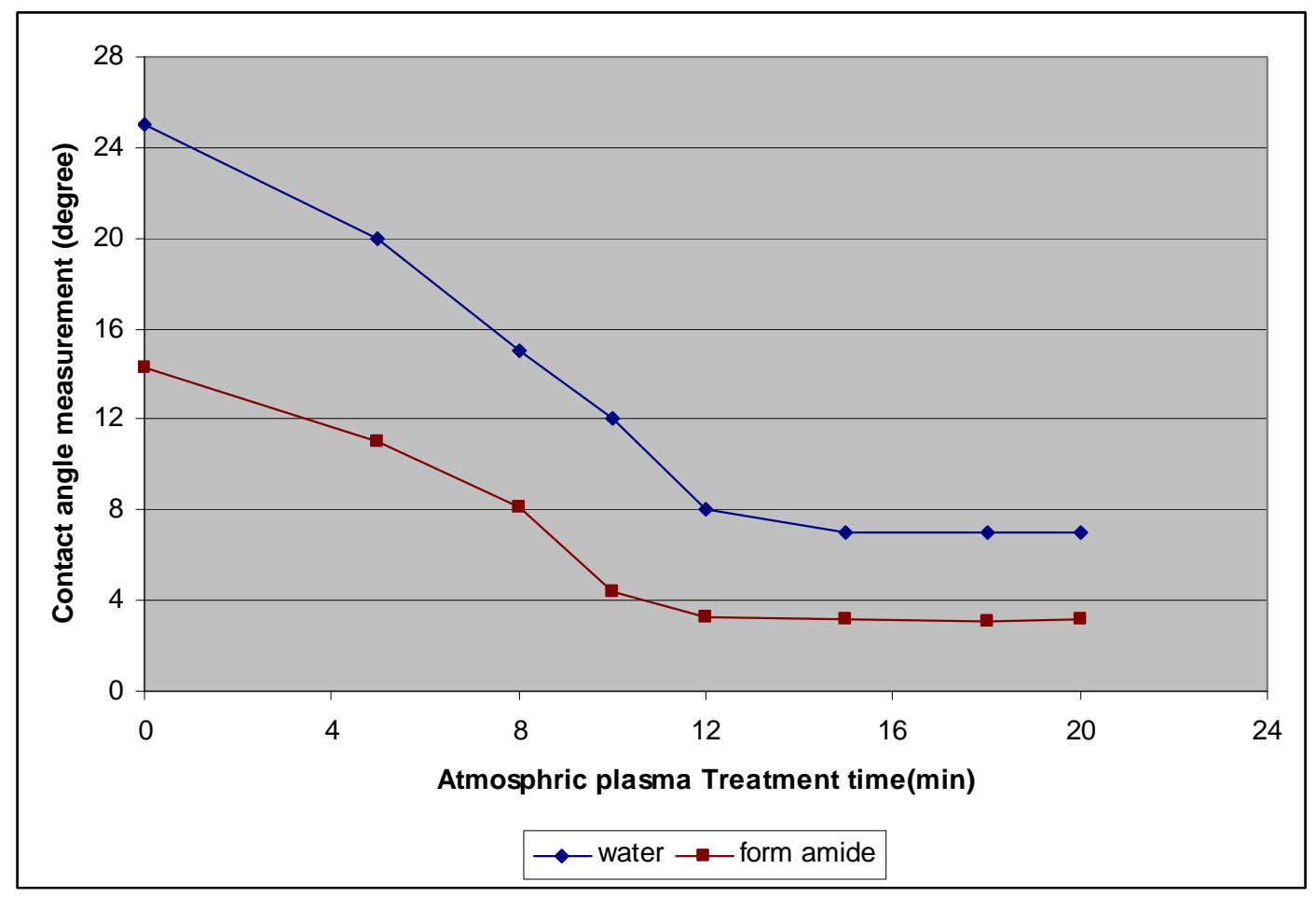

Fig. 2 Contact angle measurement with water after gritblasting + atmospheric pressure plasma treatment 


\subsection{Surface Energy of as Received, Mechanical Treated and Plasma treated Titanium samples}

The Surface energy and it's two components of unmodified titanium, surface modified titanium under 120 sec of grit blasting and surface modified titanium under 120 sec of grit blasting followed 15 minutes of exposure under atmospheric pressure plasma treatment is shown in figure 3. The figure shows that polar component of surface energy increases significantly with mechanical treatment and there is further improvement if the mechanical treatment is followed by an atmospheric pressure plasma treatment. The dispersion component decreased after both the mechanical and combined grit blasting and plasma treatment. For both treated surfaces the surface energy is mainly determined by the polar component.

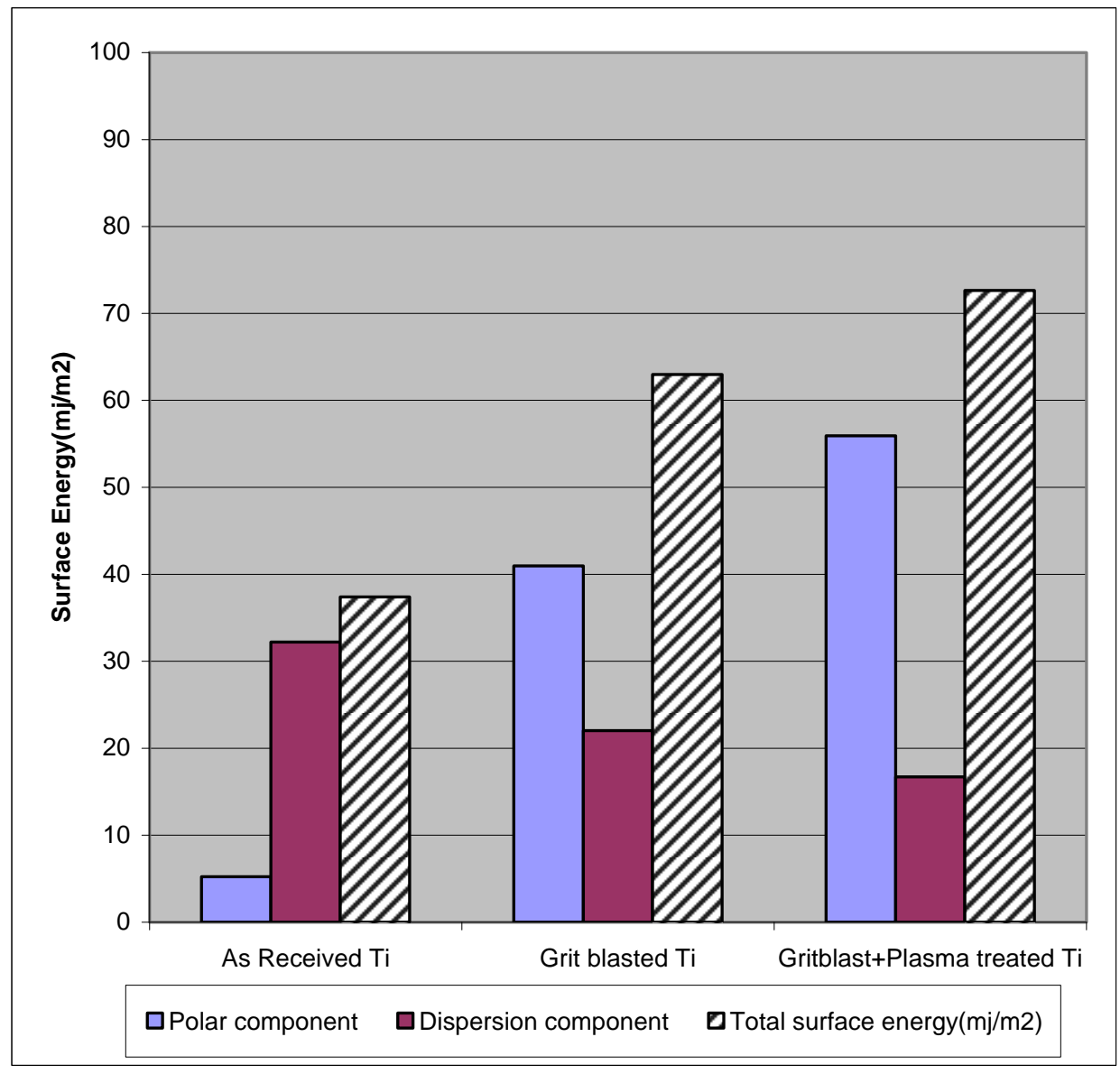

Fig.4 Surface energy of titanium sample after different surface treatments 


\subsection{Surface Roughness Analysis}

The Surface roughness of as received titanium washed with ethanol, grit blasted samples for 30, 60, 90 and $120 \mathrm{~s}$ and atmospheric pressure plasma treated samples were measured using a WYKO NT300 surface roughness profiler. The results of the surface roughness measurements are presented in figure 4 . The figure reveals that the surface roughness of titanium increases with increasing treatment time up to $120 \mathrm{sec}$ and then it saturates. The Surface roughness increases from nanometre to micrometre after grit blasting as indicated in Fig 5(a) and 5(b). When this 120 sec grit blasted titanium surface was further modified by atmospheric pressure plasma for 15 minutes, there is no further increase in surface roughness as evident from Fig 5(b).

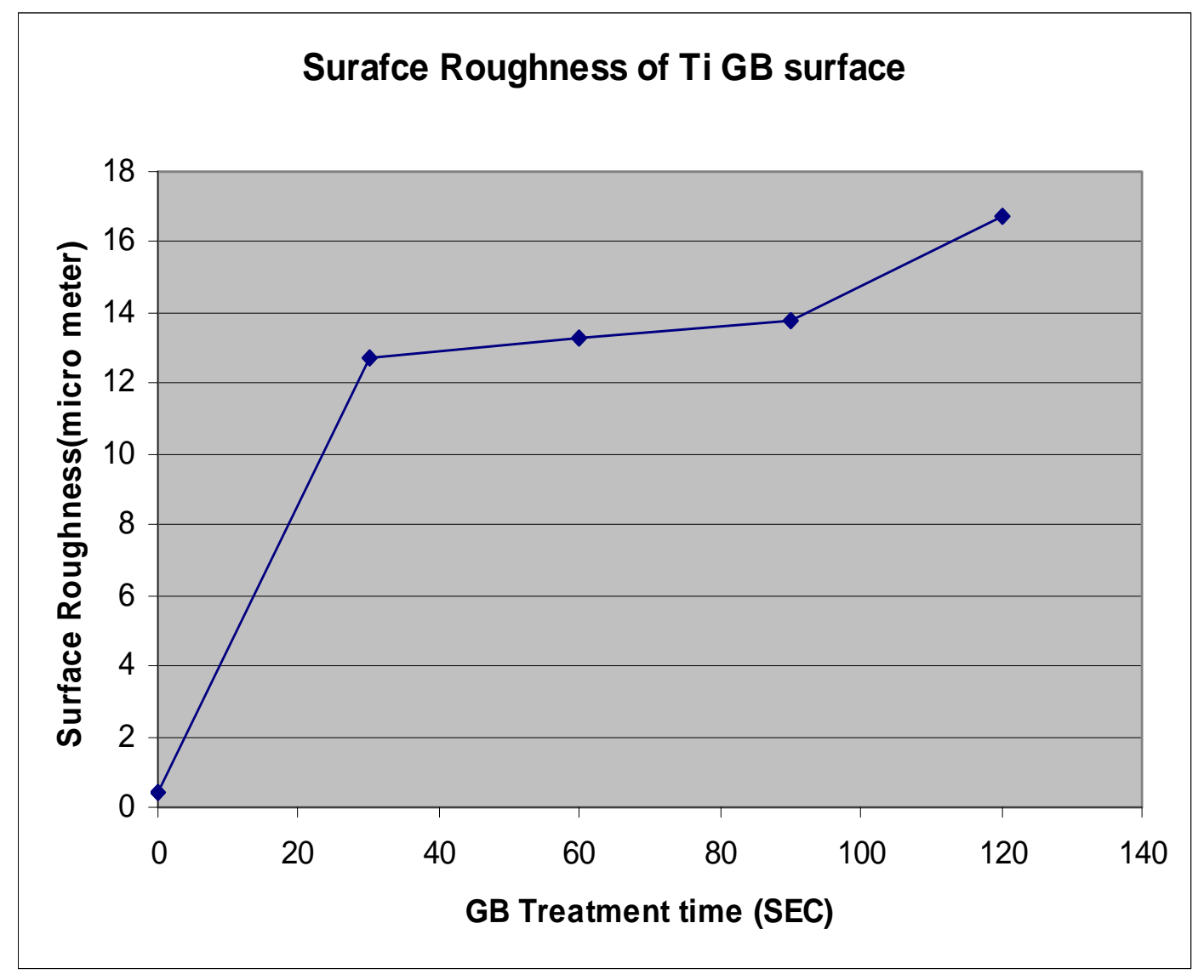

Fig. 4 Surface roughness analysis with mechanical treatment time 

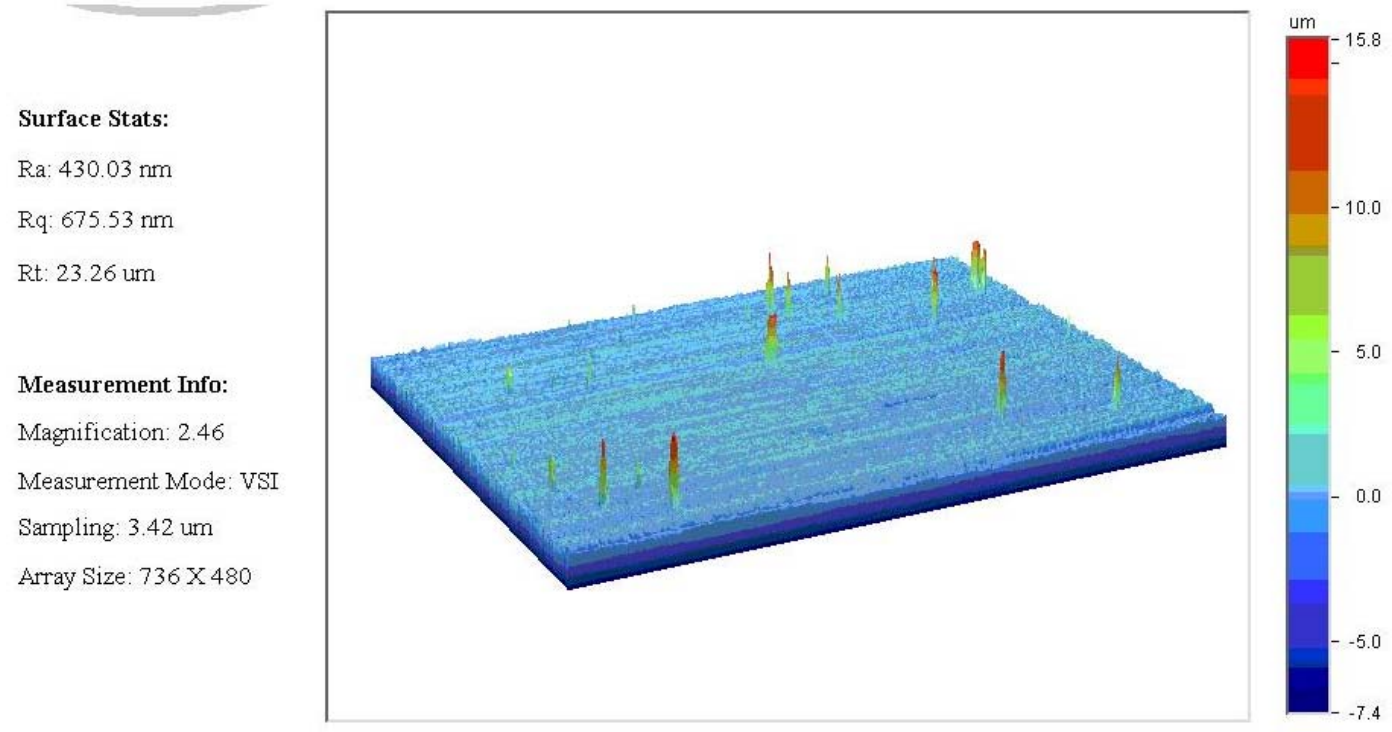

Fig. 5(a) Surface roughness As received titanium samples.

Surface Stats:

Ra: 16.72 um

Rq: 20.72 um

Rt: 125.72 um

Measurement Info:

Magnification: 20.48

Measurement Mode: VSI

Sampling: $410.16 \mathrm{~nm}$

Array Size: 736 X 480

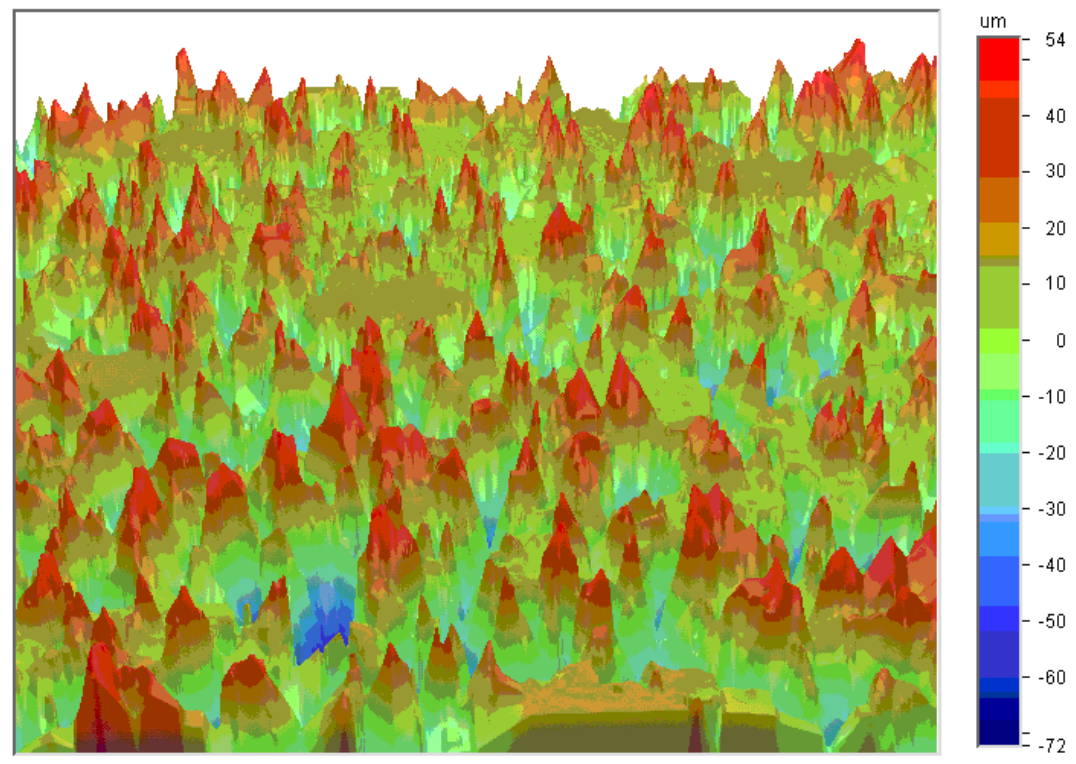

Fig. 5(b) Surface roughness after grit blasting and atmospheric plasma treatment 


\subsection{Scanning Electron Microscope Images}

As received titanium samples, grit blasted samples and grit blasted followed by atmospheric pressure plasma treated samples were analysed using SEM in order to understand the change in surface with various treatments and shown in Fig. 6 (a), 6 (b) and 6 (c) respectively.

A difference in surface roughness of as received titanium surface and grit blasted titanium surface were clearly observed in SEM images as shown in figure 6(a) and 6(b). The figure reveals that there is a remarkable increase in surface roughness after grit blasting of titanium. However, there is no distinct change in the surface roughness of titanium when it is subjected to atmospheric pressure plasma treatment as shown in Fig6(c).

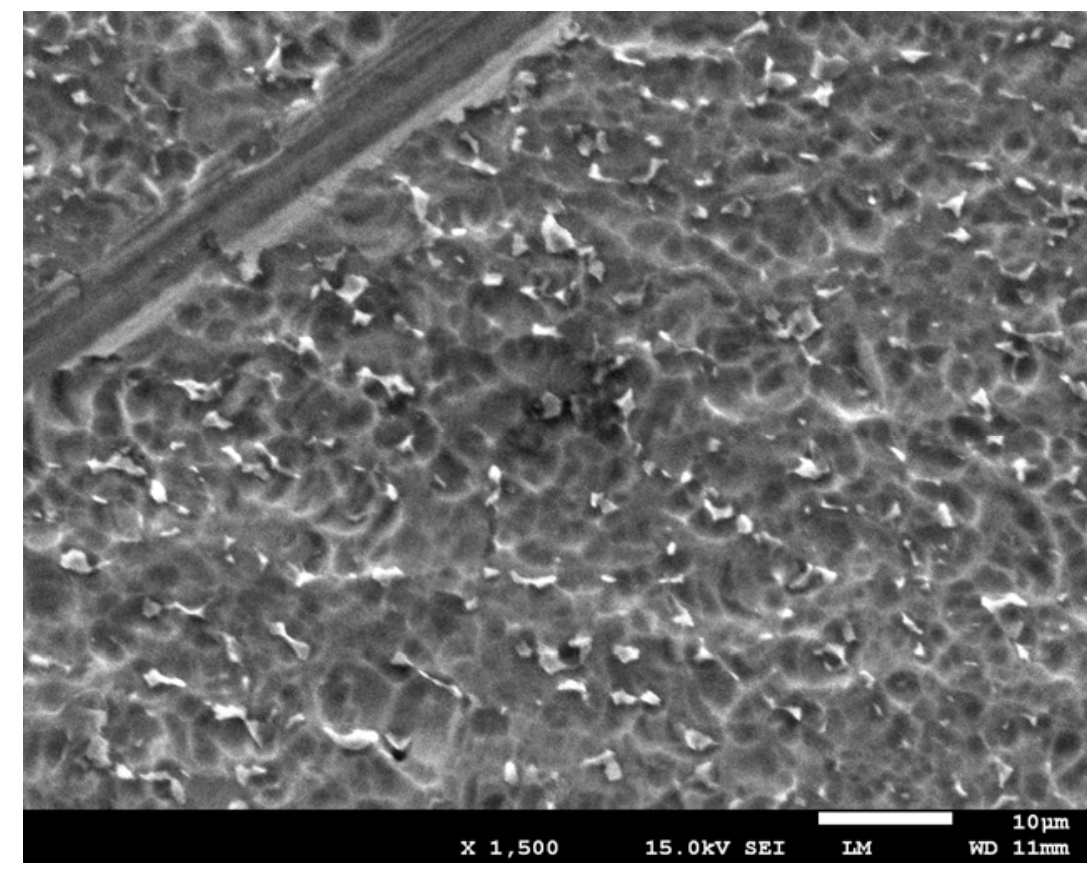

Fig. 6(a) As received titanium alloy after ultrasonic cleaning with ethanol X1500 magnification 


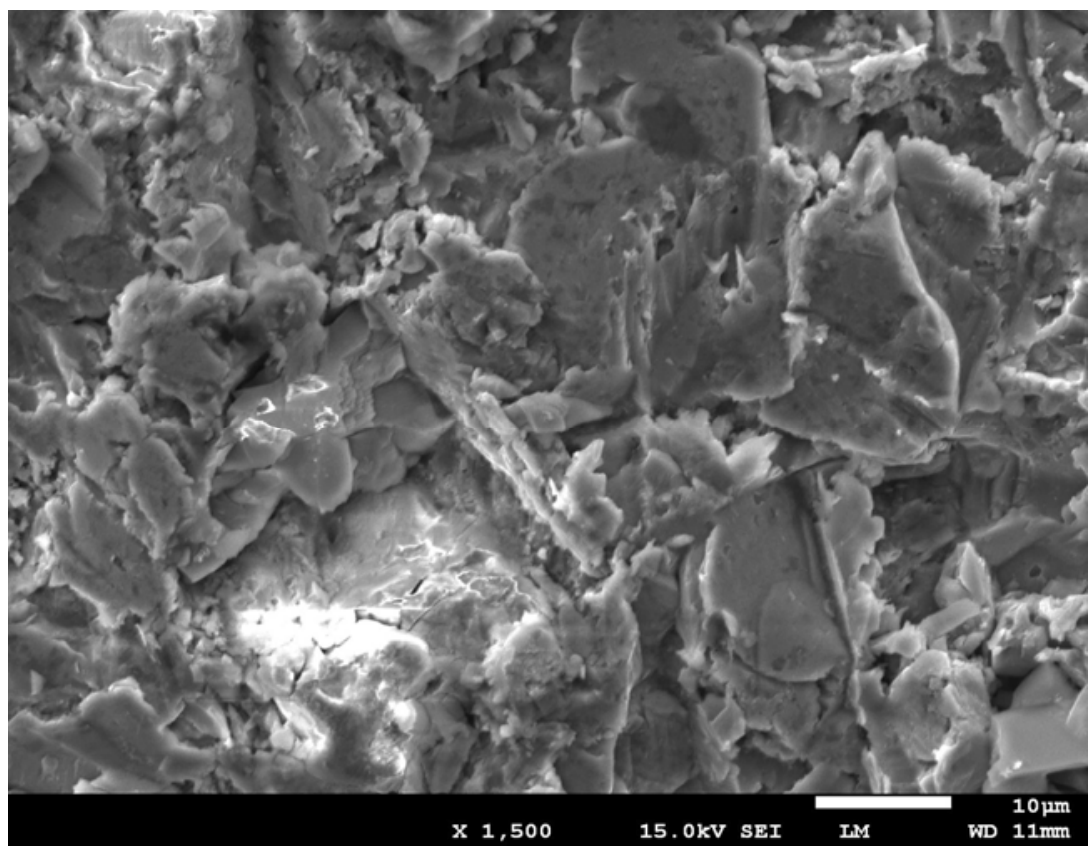

Fig. 6(b) Grit blasted with alumina and ultrasonically cleaned with ethanol X1500 magnification

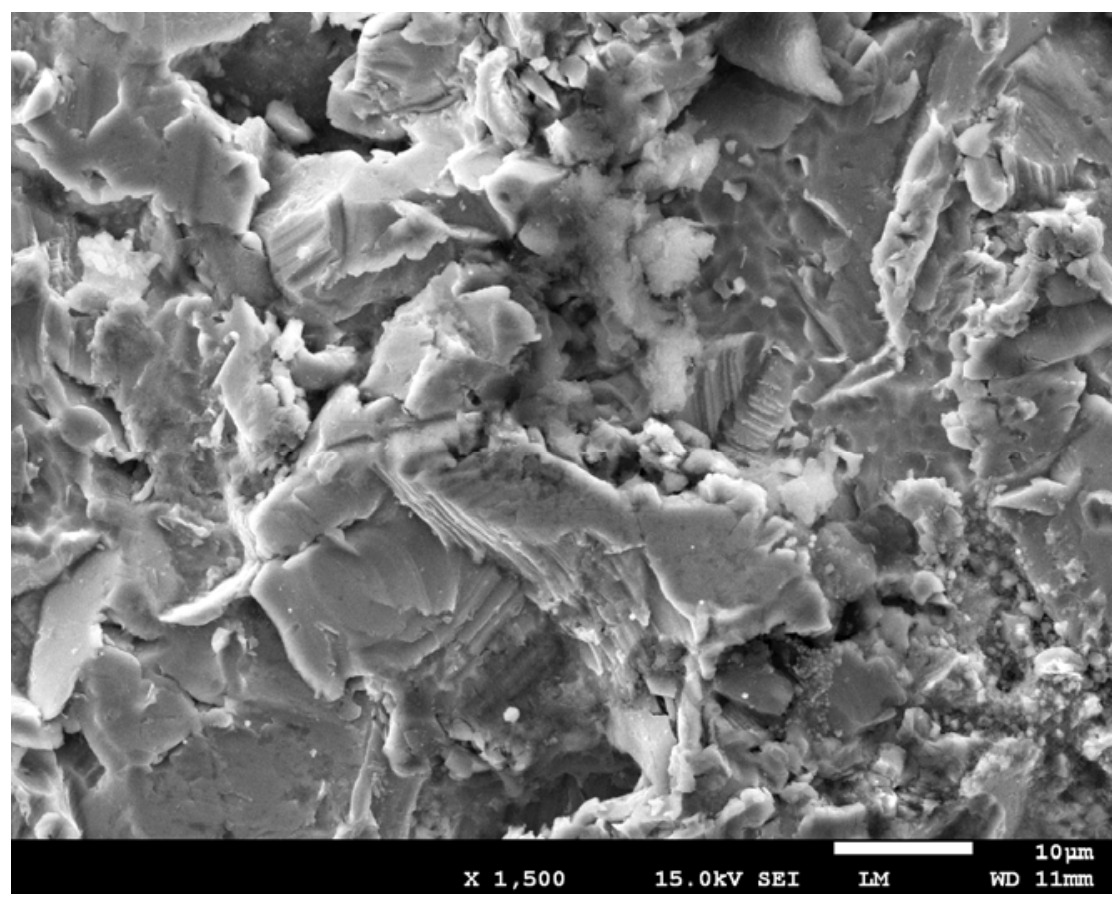

Fig. 6(c) Grit blasted, ultrasonically cleaned with ethanol and plasma treated for 20 minutes X1500 magnification 


\subsection{Lap Shear Tensile Properties of Adhesive Bonded Joints}

Tensile strength properties of single lap shear adhesive joints of titanium to titanium are shown in figure 7 . The figure reveals that the adhesive bond strength of titanium to titanium of as received titanium when bonded with high performance polyimide adhesive is 3.65 MPa. When the titanium surface is mechanically treated by grit blasting for 120 seconds, there is a considerable increase in adhesive bond strength up to $7.75 \mathrm{MPa}$. When the titanium surface is further modified by atmospheric pressure plasma, there is a further increase in adhesive bond strength up to 10.10 MPa. The Lap shear joint of as received titanium samples failed at the interface of titanium substrate and the adhesive whereas in the case of grit blasted and plasma treated samples the failure mode shifted from interface to within adhesive. Failure modes are presented in Figure 8(a) and 8(b).

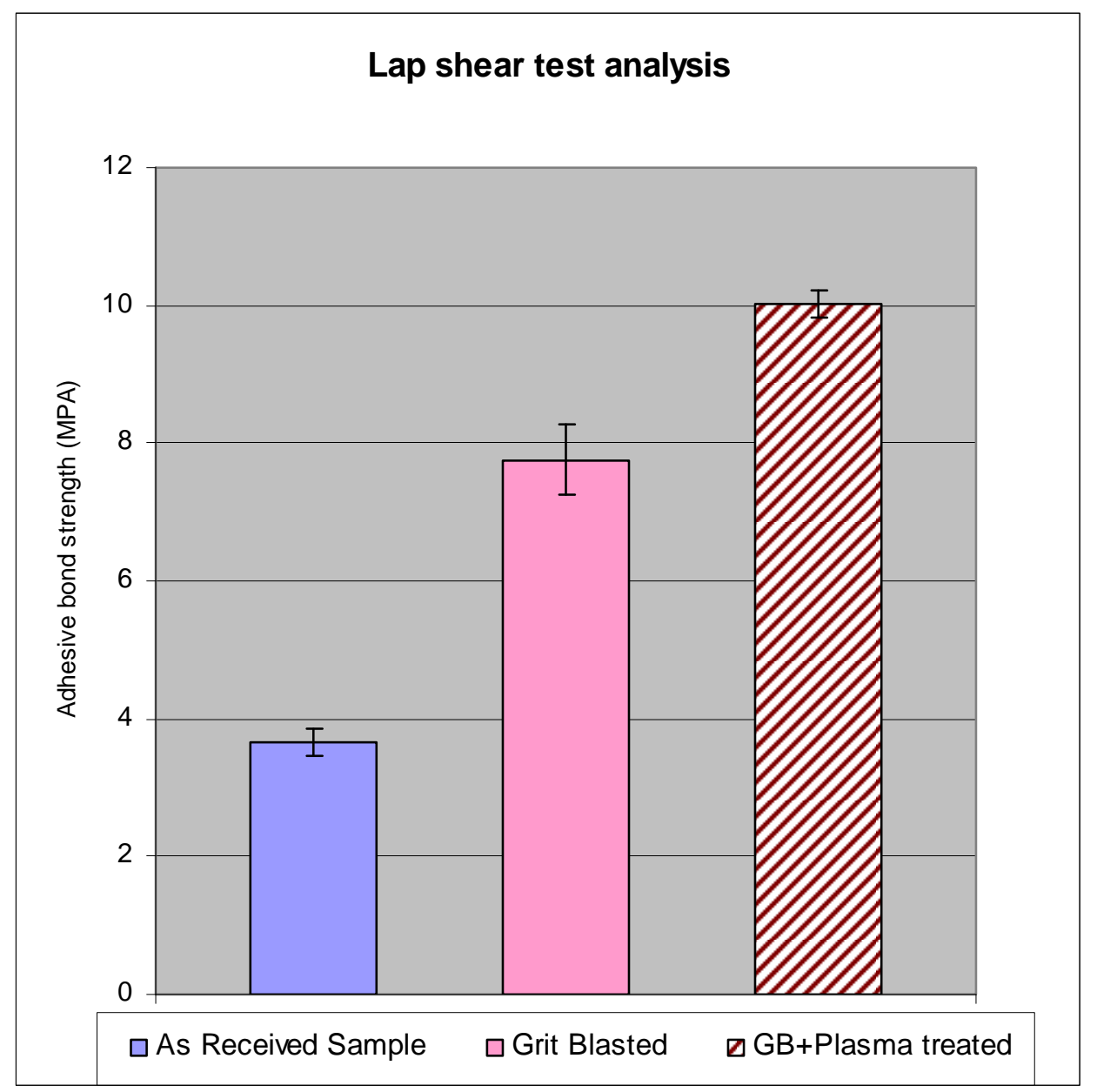

Fig. 7 Adhesive joint strength of as received titanium and surface modified titanium 


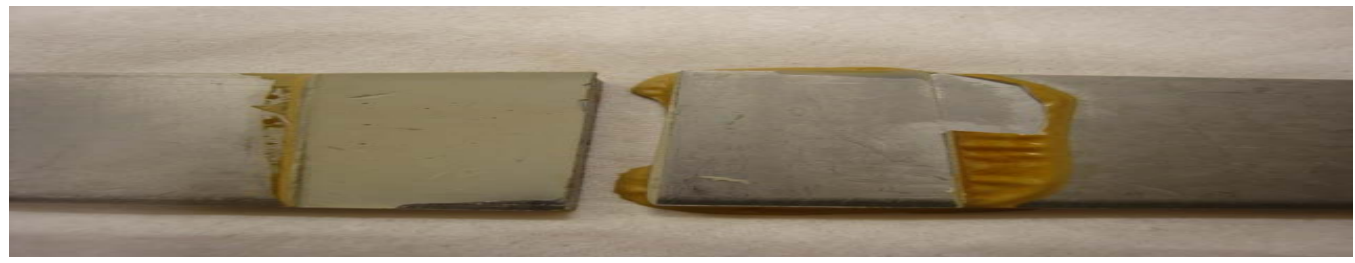

Fig 8(a) Bond failure during lap shear test at adhesive metal interface before any treatment

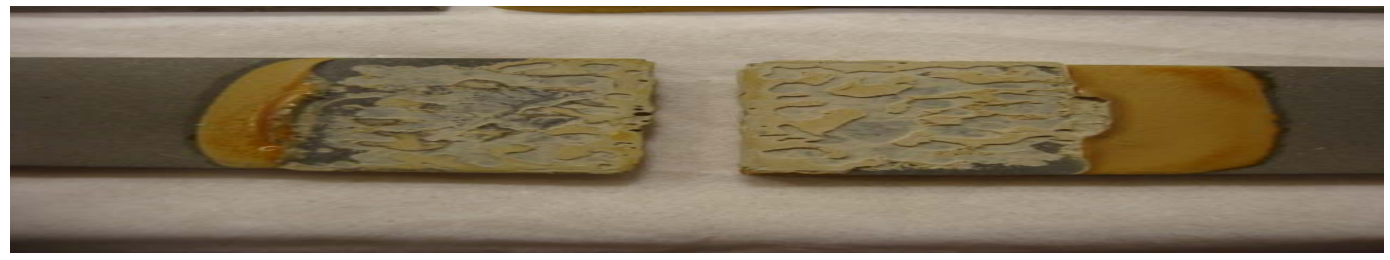

Fig 8(b) Bond failure during lap shear test within adhesive after grit blast and plasma treatment

\section{Discussion}

The present investigation was carried out to justify qualitatively and quantitatively the effect of mechanical grit blasting and atmospheric pressure plasma treatment on the surface energy of titanium alloy leading to improved adhesion characteristics of bonded joints with polyimide adhesive. The influence of surface treatment of titanium on bond strength is also studied. Mechanical grit blasting and plasma treatment for surface modification is used to produce hydrophilic surfaces on metals [13].

Untreated titanium alloy surfaces are covered with organic impurities, inhibitors, and degassing agents used during casting and rolling of the alloy. Titanium surfaces therefore do not posses the desired surface properties to form strong adhesive bonds. They are less reactive in nature due to contamination on the surface, and in general exhibit insufficient adhesive bond strength due to relatively low surface energy as shown by the contact angle and surface energy measurements [14]. Therefore, in order to improve the surface energy of titanium, the titanium surface was modified by grit blasting followed by atmospheric pressure plasma treatment. The contact angle and surface energy of grit blasted titanium improves with increase in grit blasting time. SEM analysis and surface roughness measurements with a Wyko surface roughness profiler of a Grit blasted surface indicates that the increase in macro roughness is one of main factors for the increase in surface energy which leads to an increase in over all area available for contact between adhesive and metal surface. Mechanical treatment of Titanium surface attributes to increase in area for 
adsorption of adhesive on metal surface. After grit blasting, the improvement in adhesive bond strength was confirmed by single lap shear tensile tests.

Atmospheric pressure plasma treatment on mechanically modified titanium surface, leads to much lower contact angle and ultimately further increase in surface energy which resulted in more improvement of adhesive bond strength. Electrochemical behaviour of titanium surface could be an important factor resulting in weakening the interface of titanium-adhesive[15]. XPS analysis of commercial titanium alloy carried out by Panousis et al [7] reveals that there is high percentage of carbon and silicon before plasma treatment resulting from presence of contaminated layer on titanium surface. Panousis et al performed low pressure plasma treatment on titanium surface in vacuum .XPS analysis of low pressure plasma treated surface indicates that there is an increase of percentage of oxygen and percentage of carbon and silicon decreases [7].it is expected that atmospheric pressure plasma treatment has same kind of effect on titanium surface as low pressure plasma. These results suggest that due to plasma treatment there is extensive cleaning on titanium surface resulting in increase in surface energy of titanium after plasma treatment. Due to atmospheric pressure plasma exposure, TiA6V4 surface cleaning from organic contaminants and surface functionalization take place. Further, activated oxygen radicals in the outer layer of titanium could be attributed to increase in surface energy[7]. Therefore, after atmospheric pressure plasma treatment, increase in surface energy is primarily due to formation of chemical functionalities and secondarily due to increase in surface roughness.

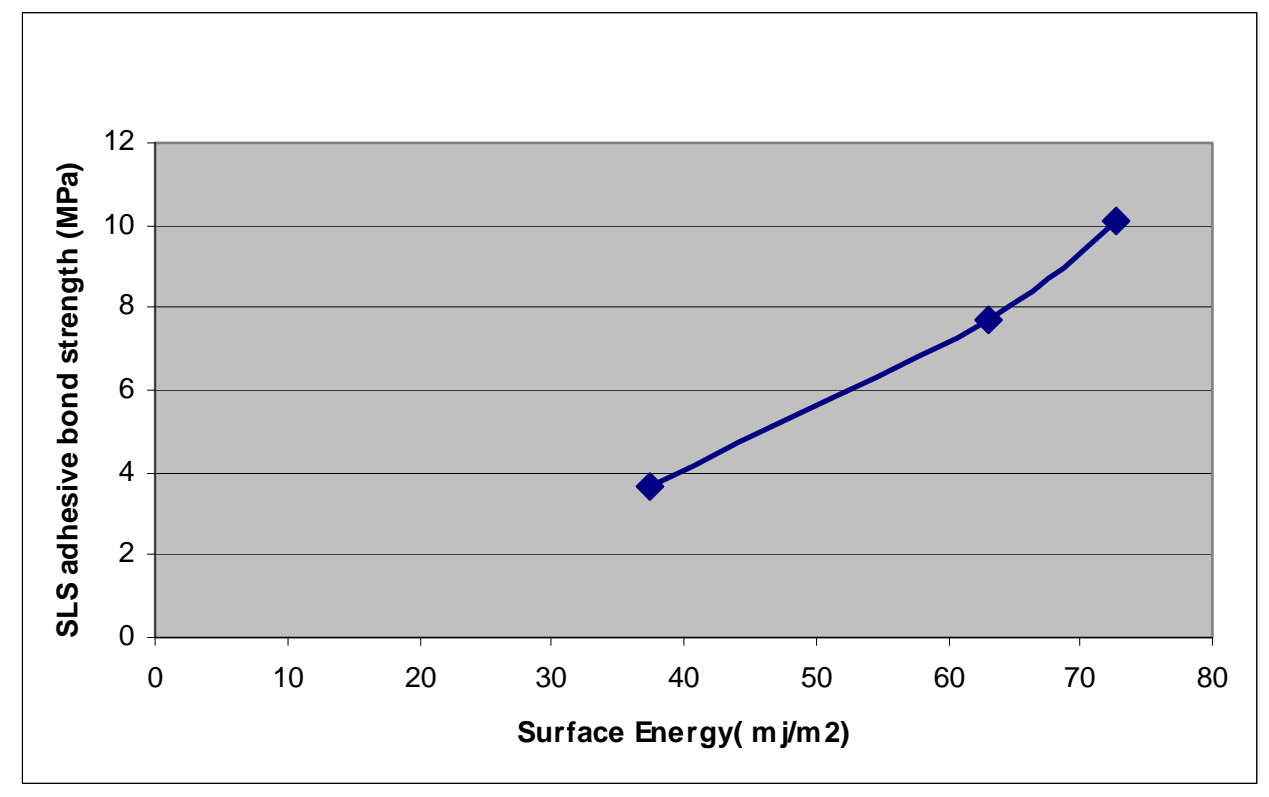

Fig. 9 SLS Adhesive bond strength vs surface energy of Titanium samples 
A relationship between surface energy and single lap shear adhesive bond is presented in Fig.9. It reveals that adhesive bond strength increase with increase in surface energy of titanium surface.

It is well established that carbonyl groups in the polymers act as the major electron donor component. Lee et al has proved that basicity of polymers strongly correlated with its carbonyl group mole fraction[16].It is expected that the carbonyl group present in the polyimide adhesive could make strong a Lewis base interaction with the titanium surface. Therefore, atmospheric pressure plasma treatment on titanium, essentially removed organic contamination followed by an increase of Lewis acid titanium oxide resulting in formation of extra chemical bonds leading to significant increase in adhesive bond strength as demonstrated in figure 7.Due to these extra chemical bonds the mode of failure also shifted from metal-adhesive interface to within adhesive during tensile test.

\section{Conclusions}

Titanium surfaces are mostly contaminated with impurities and organic materials. In order to increase the bond strength of titanium with high performance adhesive it is necessary to carry out cleaning of the surface. This can be done by mechanical treatment like grit blasting which resulted in increased adhesive bond strength due to increase in contact area between titanium and adhesive. Atmospheric pressure plasma treatment further removed organic impurities layer and is supposed to increase the amount of activated oxygen radicals at the titanium surface. These oxide radicals who are highly acidic in nature and react with carbonyl basic groups present in polyimide adhesive. It is shown that grit blasting followed by atmospheric pressure plasma treatment leads to significant increase in adhesive bond strength. Due to surface modification of titanium, locus of failure has shifted from metal polymer interface to within the adhesive.

\section{Acknowledgement:}

We gratefully acknowledge the technical support provided by Faculty of aerospace Engineering, Delft University of technology The Netherlands. 
$8 / 22 / 2011$

\section{References}

1. El-Hossary, F.M., et al., Surface modification of titanium by radio frequency plasma nitriding. Thin Solid Films, 2006. 497(1-2): p. 196-202.

2. Keohan, F.L.a.H., B. J. in Proceedings of the 21st Annual meeting of the Adhesion Society 1998. Savannah, Georgia.

3. The Boeing Company, BOEING-Puget sound's environmentally complaint sol-gel surface treatments for metal bonding: Box 516, St. Louis, MO 63166.

4. Molitor, P. and T. Young, Adhesives bonding of a titanium alloy to a glass fibre reinforced composite material. International Journal of Adhesion and Adhesives, 2002. 22(2): p. 101-107.

5. Bhowmik, S., et al., High-performance nanoadhesive bonding of titanium for aerospace and space applications. International Journal of Adhesion and Adhesives, 2009. 29(3): p. 259-267.

6. Gutowski, T. The mechanics of composite deformation during the manufacturing process. in First Conference of Composite Materials. American Society for Composites. 1986. Dayton, $\mathrm{OH}$.

7. Panousis, E., et al., Titanium alloy surface treatment using an atmospheric plasma jet in nitrogen pulsed discharge conditions. Surface and Coatings Technology, 2007. 201(16-17): p. 7292-7302.

8. $\quad$ G.W.Critchcow, D.M.B., Int J Adhes Adhesives, 1995. 15: p. 161.

9. E.McCafferty, LewisAcid/Lewis Base effects in corrosion and polymer Adhesion at Aluminium surfaces. Journal of the Electrochemical society, 2003. 150(7): p. B342B347.

10. J. van den Brand, O. Blajiev, P. C. J. Beentjes, H. Terryn, and J. H. W. de Wit,, Interaction of Ester Functional Groups with Aluminum Oxide Surfaces Studied Using Infrared Reflection Absorption Spectroscopy. LangMuir Article, 2004. 20: p. 6318-6326.

11. Comyn, J., Contact angles and adhesive bonding. International Journal of Adhesion and Adhesives, 1992. 12(3): p. 145-149.

12. D.E.Packham, ed. Handbook of Adhesion. 2nd ed. 2005, John Wiley \& Sons Ltd.: West Sussex PO19 8SQ.England. p.9.

13. M.C.Kim, D.K.s.H.S.S., Surface modification for hydrophilic property of stainless steel treated by atmospheric pressure plasma jet. Surface and Coatings Technology, 2003. 171: p. 312-216.

14. Dominic J. Varacalle, J., Donna Post Guillen, Douglas M. Deason, William Rhodaberger, and Elliott Sampson, Effect of Grit-Blasting on Substrate Roughness and Coating Adhesion. Journal of Thermal Spray Technology, 2006. 15(3): p. 7.

15. Bhowmik, S., et al., Durability of adhesive bonding of titanium in radiation and aerospace environments. International Journal of Adhesion and Adhesives, 2006. 26(6): p. 400-405.

16. Jung-Hyun Lee, B.J.R.T., Tae Hyun Bae and J Carson Meredith, Role of Lewis Basicity and van der Waals Forces in adhesion of silica MFI Zeolites (010) with polyimides. LangMuir Article, 2009. 25(16): p. 9101-9107. 